\title{
Bimalleolar pathological fracture in a patient with chronic tophaceous gout
}

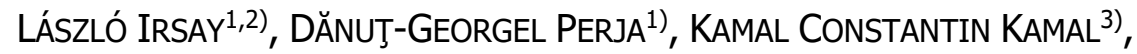 \\ MAGDALENA RODICA TRĂISTARU ${ }^{4}$, DiANA KAMAL ${ }^{5}$, VIORELA MIHAELA CIORTEA ${ }^{1,2)}$,

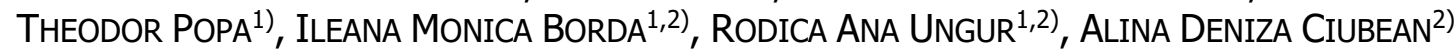 \\ 1) Department of Physical and Rehabilitation Medicine, Clinical Rehabilitation Hospital, Cluj-Napoca, Romania \\ ${ }^{2)}$ Department of Physical and Rehabilitation Medicine, Iuliu Haţieganu University of Medicine and Pharmacy, \\ Cluj-Napoca, Romania \\ 3) Department of Family Medicine, University of Medicine and Pharmacy of Craiova, Romania \\ 4) Department of Physical and Rehabilitation Medicine, University of Medicine and Pharmacy of Craiova, \\ Romania \\ 5) Department of Physical and Rehabilitation Medicine, Sama Clinic, Craiova, Romania
}

\begin{abstract}
Gout is one of the most common types of inflammatory arthritis. Four stages have been described in its evolution: asymptomatic hyperuricemia, acute gouty arthritis, intercritical gout and chronic tophaceous gout. Monosodium urate crystals deposits are more frequently encountered in hyaline cartilage, tendon sheaths, articular bursae, bone epiphysis, synovium, and skin. Pathological fractures that occur at the formation sites of gout tophi have been rarely described in gout. Bimalleolar fractures often occur following a trauma. We present the case of a 56-year-old patient, diagnosed with chronic gout for over 12 years, with tophi in the upper and lower limbs, who presented accusing mechanical pain in the left tibio-tarsal joint and impaired function of the hands. The clinical examination showed bilateral multiple destructive gout tophi in the hands, forearms, elbows, feet, ankles, and lower legs, while the left ankle exhibited an important varus deformity. Computed tomography examination of the left ankle showed multiple gout tophi with bone erosions and a bimalleolar pathological fracture. The patient underwent pharmacological treatment, physical therapy and the ankle was immobilized in a knee-ankle-foot orthosis, with subsequent orthopedic surgical treatment for fracture fixation, to improve functional status. We have chosen to present this case given the multiple disabilities of the patient, which have improved following the rehabilitation treatment.
\end{abstract}

Keywords: gout tophi, fracture, bone erosions, rehabilitation.

\section{a Introduction}

Gout is a chronic condition, characterized by the formation of deposits of monosodium urate crystals in the joints or soft tissue. It is one of the most common types of inflammatory arthritis. From a clinical point of view, it is characterized by four progressive stages: asymptomatic hyperuricemia, acute gouty arthritis, intercritical gout and, if left untreated, chronic tophaceous gout $[1,2]$. The deposition of monosodium urate crystals is more frequently encountered in the synovium, hyaline cartilage, joints, periarticular soft tissues, and it leads to the development of bone erosions [1, 3, 4].

Intraosseous tophi are a rare presentation, but if they occur, they have a subchondral bone and metaphyseal location, and may lead to pathological fractures following minor trauma or even in the absence of a traumatic event $[4,5]$. Long-term complications include joint damage and renal stones, and it is associated with an increased prevalence of chronic renal disease. In advanced stages, gout is associated with severe disability, mainly if complicated by the presence of bone fractures [1].

Biologically, hyperuricemia is defined as a uric acid level in the blood that exceeds its physiological solubility limit of $6.8 \mathrm{mg} / \mathrm{dL}$, elevated values above this threshold leading to the formation and deposition of monosodium urate crystals [6]. Uric acid is the final product of purine metabolism and excess consumption of purine rich foods and alcohol results in hyperuricemia [3,6]. Most of the uric acid is excreted by the kidneys and a smaller proportion is eliminated through the gastrointestinal tract. A decreased renal excretion causes uric acid accumulation, with subsequent deposition and tophi formation $[6,7]$.

Gouty tophi represent a chronic inflammatory granulomatous reaction secondary to the presence of monosodium urate crystals $[8,9]$, with various pro-inflammatory factors being expressed, among them interleukin (IL)-1 $\beta$, IL-6, and tumor necrosis factor-alpha (TNF- $\alpha$ ). These cytokines can stimulate osteoclasts and chondrocytes, which are implicated in bone erosions [6, 8-10]. Furthermore, monosodium urate deposits decrease osteoblastic activity, contributing to the production of bone lesions and preventing healing, resulting in bone fragility and fractures [9].

The treatment of asymptomatic hyperuricemia and gout consists of patient education, hygienic-dietary regime, steroidal and nonsteroidal anti-inflammatory medication, urate lowering therapy and means of medical rehabilitation

This is an open-access article distributed under the terms of a Creative Commons Attribution-NonCommercial-ShareAlike 4.0 International Public License, which permits unrestricted use, adaptation, distribution and reproduction in any medium, non-commercially, provided the new creations are licensed under identical terms as the original work and the original work is properly cited. 
through which the effects of disability are minimized, and attempts are made to increase functionality.

\section{Aim}

We present a rare case of pathological fracture due to gout tophi, which imposed a diagnostic challenge, and showed the importance of multidisciplinary management in such conditions. Usually, gout is not associated with an increased risk of fracture, but our patient had fractures of the upper and lower limbs and multiple disabilities, which have improved following the complex rehabilitation treatment.

\section{ㅁ Case presentation}

A 56-year-old patient with known chronic tophaceous gout and a history of surgical removal of bilateral elbow and dorsal forearm tophi, surgical neck fracture of the left humerus (August 2018), high blood pressure and duodenal ulcer surgery (2002) was referred to the Department of Physical and Rehabilitation Medicine within the Clinical Rehabilitation Hospital, Cluj-Napoca, Romania, in September 2019, complaining of mechanical left ankle pain, functional impairment of the hands and impaired joint mobility of the left shoulder.

The physical examination revealed multiple gout tophi on fingers, hands (Figure 1, A and B), forearms and elbows and an inferior subluxation of the left shoulder with associated pain and limited range of motion in all planes (Table 1).

The disabilities of the arm, shoulder and hand (DASH) score at admission was 70 points.

The lower extremities exhibited multiple gout tophi on toes, soles, heels, Achilles' tendons and calves, and a varus deformity of the left ankle was noted (Figure 2, A-D).
Table 1 - Active range of motion in the left shoulder

\begin{tabular}{cl}
\hline Flexion & $75^{\circ}$ \\
\hline Extension & $45^{\circ}$ \\
\hline Abduction & $75^{\circ}$ \\
\hline Internal rotation & $75^{\circ}$ \\
\hline External rotation & $50^{\circ}$ \\
\hline
\end{tabular}
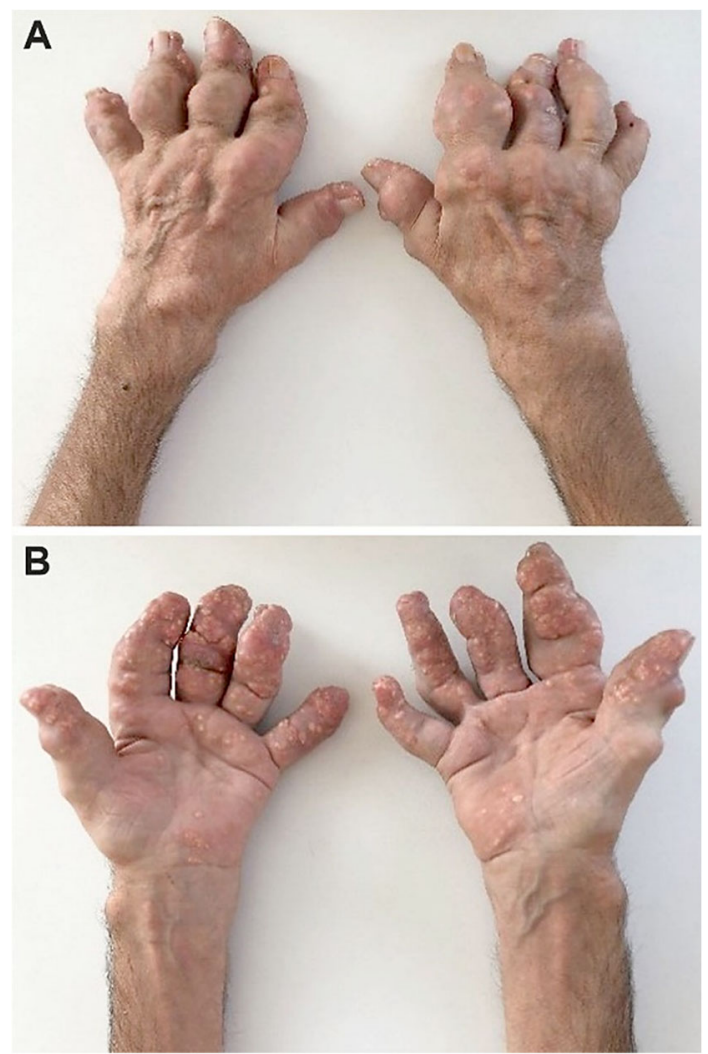

Figure 1 - Gout tophi on fingers and hands: (A) Hands in prone posture; (B) Hands in supine posture.
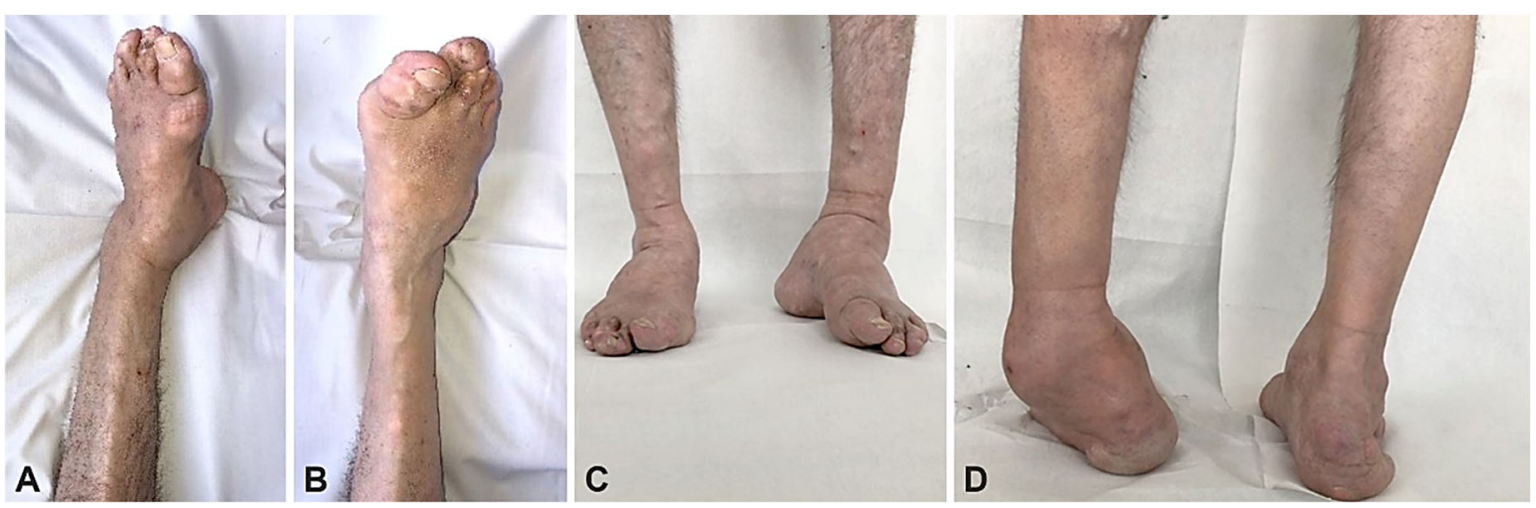

Figure 2 - Gout tophi on lower extremities: $(A-C)$ Anterior view showing multiple gout tophi on toes, soles, and calves;

(D) Posterior view - varus deformity of the left ankle and multiple gout tophi on heels and Achilles' tendons.

Laboratory data revealed: erythrocyte sedimentation rate (ESR) $20 \mathrm{~mm} / \mathrm{h}$; triglycerides $255 \mathrm{mg} / \mathrm{dL}$; alkaline phosphatase (ALP) $137 \mathrm{U} / \mathrm{L}$; creatinine $1.2 \mathrm{mg} / \mathrm{dL}$; urea $52 \mathrm{mg} / \mathrm{dL}$; creatinine clearance (Cockcroft-Gault equation) $68 \mathrm{~mL} / \mathrm{min}$; glomerular filtration rate (GFR) [Chronic Kidney Disease Epidemiology Collaboration (CKD-EPI) equations for GFR] $67 \mathrm{~mL} / \mathrm{min} / 1.73 \mathrm{~m}^{2}$ - CKD stage 2, according to Kidney Disease Outcomes Quality Initiative (KDQOI) Guidelines; urinary uric acid $120 \mathrm{mg} / 24 \mathrm{~h}-$ decreased excretion.

The patient had a very low fluid intake (approximately
$500 \mathrm{~mL} / 24 \mathrm{~h})$ and, after one week of oral hydration (2000 $2500 \mathrm{~mL} / 24 \mathrm{~h})$, the repeated laboratory data showed a slight improvement regarding renal function (creatinine clearance $73 \mathrm{~mL} / \mathrm{min}$, GFR $72.1 \mathrm{~mL} / \mathrm{min} / 1.73 \mathrm{~m}^{2}$ ) and renal excretion of uric acid (urinary uric acid $236 \mathrm{mg} / 24 \mathrm{~h}$ ).

$\mathrm{X}$-rays of both hands showed severe destructive bone changes of the phalanges, metacarpophalangeal and radiocarpal joints (Figure 3).

The computed tomography (CT) examination of the left foot revealed changes in bone structure, with subchondral tibio-tarsal and intertarsal bone erosions, as well 
as a bimalleolar fracture, soft tissue infiltration and the presence of gout tophi (Figure 4, A and B).

Because gait and ambulation were very painful, and the patient presented a high risk of severe complications like necrosis or vascular ruptures, orthopedic surgery was advised. An internal fixation with a centro-medullary rod and nails was carried out, with good functional outcome (Figure 5, A and B).

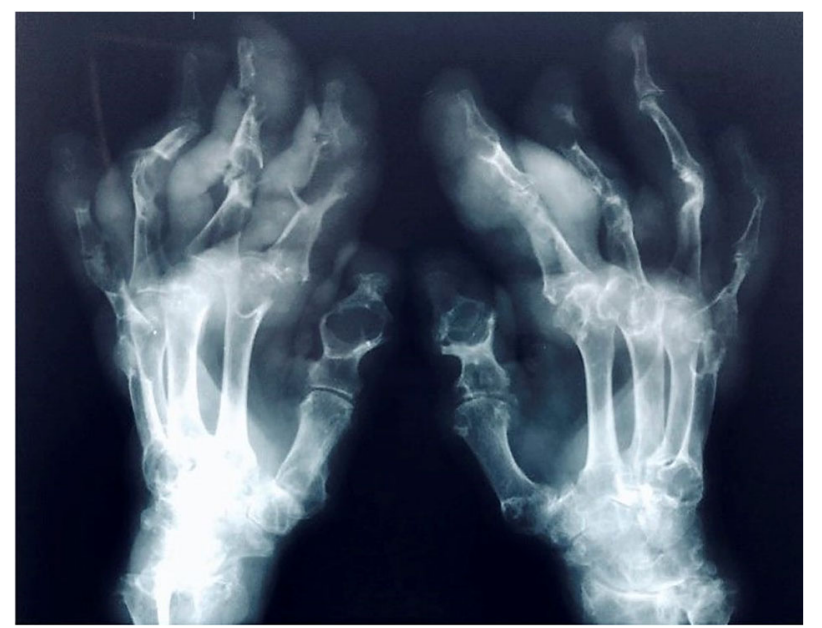

Figure 3 - Hand X-ray, bilateral antero-posterior view showing severe destructive bone changes of the phalanges, metacarpophalangeal and radio-carpal joints.
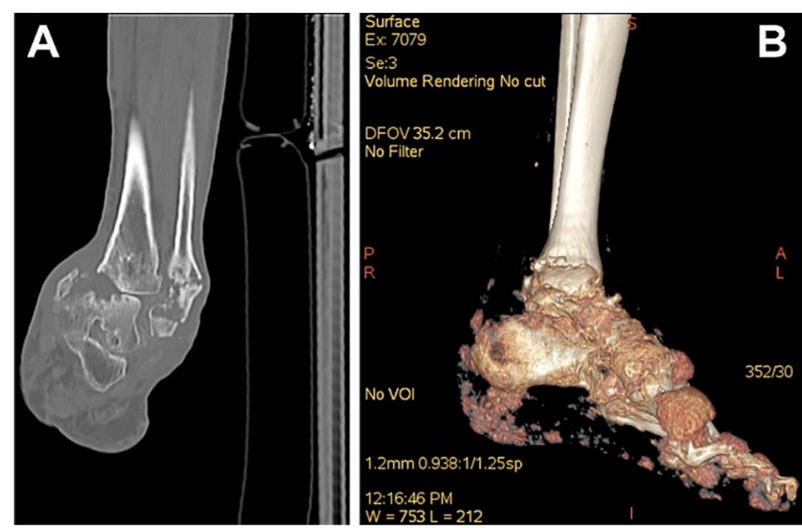

Figure 4 - Left foot CT: (A) Coronal plane bone window shows bimalleolar fracture; (B) 3D VRT shows the presence of gout tophi. 3D: Three-dimensional; CT: Computed tomography; VRT: Volume rendering technique.
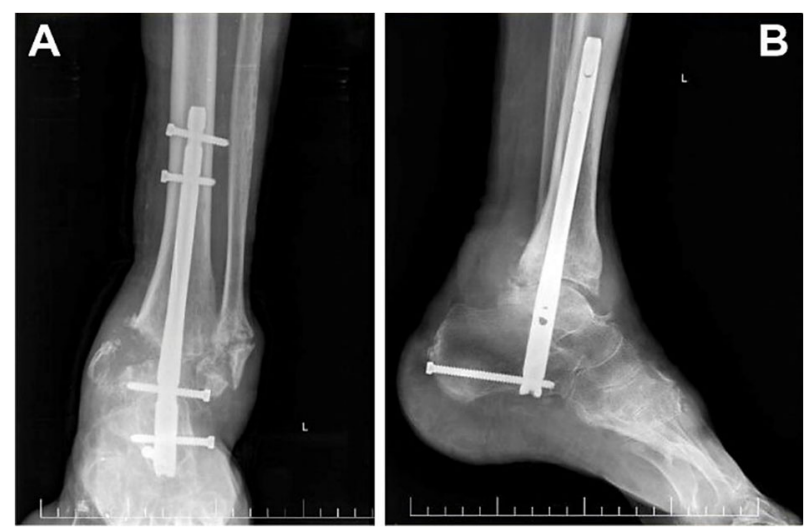

Figure 5 - Left foot X-ray: (A) Antero-posterior view after the surgery; (B) Lateral view after the surgery. Internal fixation with a centro-medullary rod and nails.
After removal of the tophi from the elbows and dorsal forearms, the excised tissue was fixed in $10 \%$ neutral buffered formalin, then washed with water, dehydrated, included in paraffin blocks, and sectioned, followed by histological and immunohistochemical (IHC) analysis of the slides obtained. Standard Hematoxylin-Eosin (HE) staining was used for histological analysis (Figure 6), and anti-cluster of differentiation 68 (CD68) antibody (Dako, clone PG-M1, 1:100 dilution, antigen retrieval seven cycles citrate buffer) was used for IHC analysis (Figure 7, A and B).

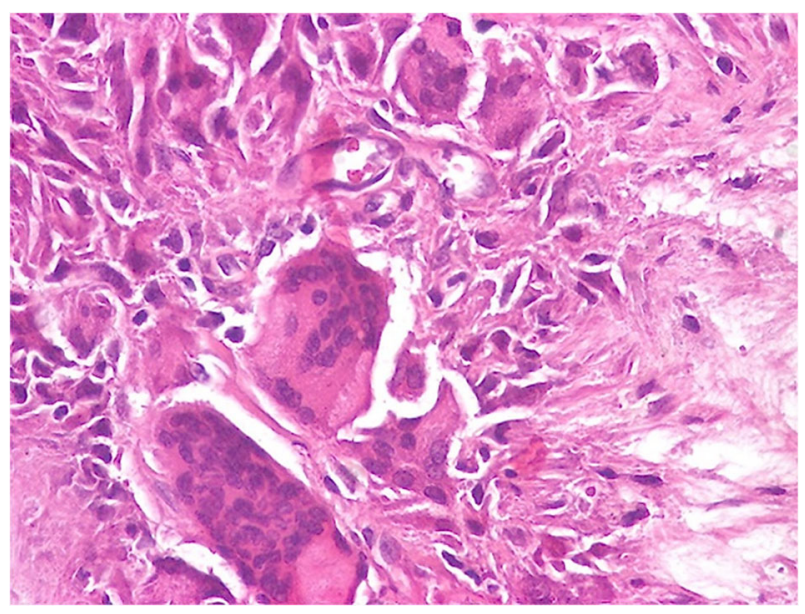

Figure 6 - Urate deposits bounded by enlarged multinuclear macrophage. Hematoxylin-Eosin (HE) staining, $\times 200$.

We noticed on all the slides obtained from our patient that the tophus contained a monosodium urate core surrounded by inflammatory cell complexes, most of them being multinucleated and having very large dimensions and adjacent fibrovascular tissue. The monosodium urate crystals of are water-soluble and thus are dissolved during routine tissue processing, so the inside of the tophus has an anucleated, necrotic appearance. Cells that bordered the anucleated nucleus of tophus showed an intense positive reaction to $\mathrm{CD} 68$, which proves that they are inflammatory cells. In the fibrovascular area, we also noticed cells that showed a positive immunoreaction, but weaker and in small numbers.

After the surgery, gait and ambulation was possible with an ankle-walker orthosis. We considered useful the use of an assistive device, such as a cane for six weeks after the surgery to improve safety, gait and to reduce stress of the affected joint. The rehabilitation treatment consisted in soft tissue massage, stretching, strengthening, gait and balance exercises. Other therapeutic methods used were thermotherapy, ultrasound, electrical stimulation, and laser, to decrease pain and increase the functionality of the involved joints. Additionally, conservatory treatment was included, consisting of hydration with 2000-2500 mL liquids per day, a hypourinic diet and hypouricemia medication. Discharge DASH score was 63.3 points, showing an improvement in the functionality of the upper limbs.

\section{ㅁ Discussions}

Monosodium urate deposits are commonly found in soft tissues, ligaments, or skin. Tophi have an organized structure, with a crystalline center consisting of a collection 
of monosodium urate crystals, surrounded by a crown in which multinucleate giant cells, such as histiocytes,

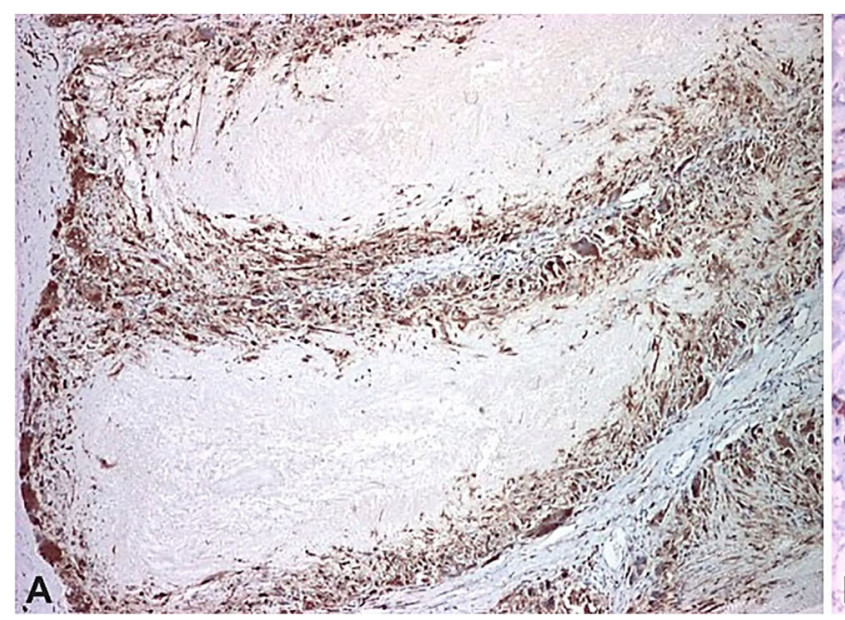

Figure 7 - (A and B) Intense positive immunoreaction in giant macrophage cells. Anti-CD68 antibody immunostaining: (A) $\times 40$; (B) $\times 100$. CD68: Cluster of differentiation 68 .

In gout pathogenesis, the activation of the inflammasome, the caspase system and IL-1 synthesis play a central role. But the main event occurring in gout inflammation is the activation of inflammatory cells by the monosodium urate crystals. According to Danger's theory, the immune system constantly scans the cells and tissues to keep everything fully and normally functioning. If certain foreign substances are liberated from the cells, the Danger system detects them immediately and provides a response. These signaling molecules are called Danger-associated molecular pattern (DAMP) $[12,13]$.

It was previously demonstrated that monosodium urate crystals represent a DAMP signal for Toll-like receptors (TLR2 and TLR4). Also, besides natural immunity, adaptative immunity is also activated. The trigger for the adaptative immune response is the dendritic cells that carry the antibody of T-cells from lymphatic nodules. The monosodium urate crystals, as DAMP, activate de dendritic cells and other immune reactions, creating an immunity cascade, where also the monocyte/macrophage, fibroblasts from the synovium and neutrophilic granulocytes are activated.

The proinflammatory cytokines that are synthetized (IL-1, IL-6, IL-8, TNF- $\alpha$ ) create another immunity cascade, where prostaglandins, leukotrienes and reactive oxygen species take part. Cytokines induce the expression of adhesion molecules, like vascular cell adhesion molecule-1 (VCAM-1) and E-selectin, on vascular endothelial cells, that facilitate the adherence of leukocytes to the endothelium and their release in the vascular system. The mediator of the sterile inflammation associated to cell necrosis on crystal induction is IL-1. This cytokine has two isoforms, IL- $1 \alpha$ and IL- $1 \beta$, but that bind to the same IL- 1 receptor. Also, caspase- 1 is activated by a cascade reaction. There are several factors that can inhibit the immunity cascade and inflammation. Phagocytosis induced by the crystals can be inhibited by apolipoprotein $\mathrm{E}$ and $\mathrm{B}$. Also, inhibition of the synovial inflammation is facilitated by the antiinflammatory cytokines, like tumor growth factor-beta (TGF- $\beta$ ), IL-10 and peroxisome proliferator-activated receptor gamma (PPAR- $\gamma$ ), and by the apoptotic cells and the exhaustion of proinflammatory mediators $[13,14]$. fibroblasts, lymphocytes, and a fibrovascular area are most commonly present [11].

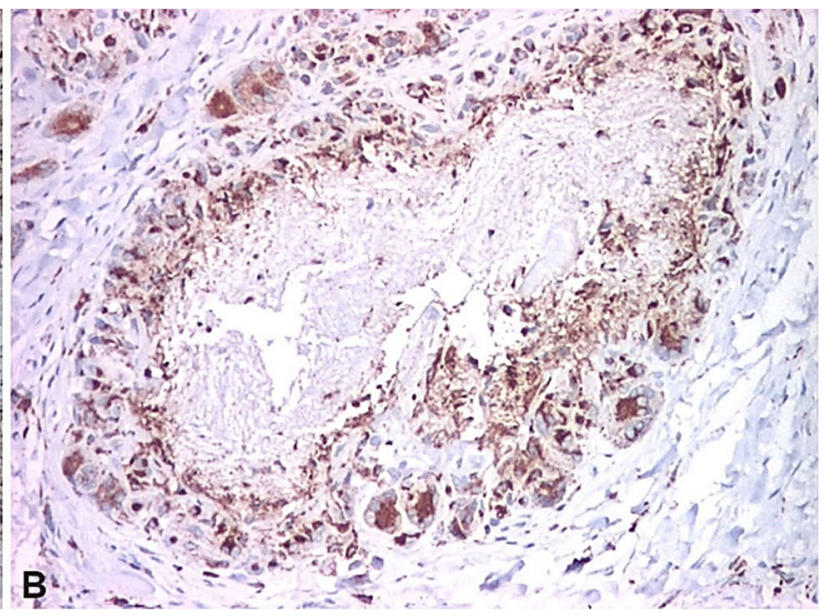

In several histological studies, cells from the outside of the tophus showed a positive immunoreaction for CD68, indicating their monocytic/macrophage nature $[10,15]$. CD68 is a protein highly expressed by cells in the monocyte lineage (monocytic phagocytes, by circulating macrophages, and by tissue macrophages). We used the anti-CD68 antibody precisely to highlight the immunoinflammatory reaction in the coronary area of gouty tophus, an aspect studied and noticed by other authors, as well as the presence of inflammatory cells in small numbers and with a much weaker reaction that can be found disseminated in the adjacent fibrovascular area [16].

\section{a Conclusions}

We presented a patient with poorly controlled gout, with severe disability after a bimalleolar fracture due to intraosseous tophi. After orthopedic surgery and conservative treatment, the quality of life of the patient increased, as gait and ambulation were restored. But, due to coronavirus disease 2019 (COVID-19) pandemic and lockdown, clinical follow-up and additional laboratory tests could not be performed.

\section{Conflict of interests}

The authors declare that there is no conflict of interests regarding the publication of this paper.

\section{References}

[1] Chadwick CM, Saxby TS. Gout associated with fracture of the fifth metatarsal shaft: a case report. Foot Ankle Int, 2011, 32(8):826-829. https://doi.org/10.3113/FAI.2011.0826 PMID: 22049871

[2] Chhana A, Dalbeth N. The gouty tophus: a review. Curr Rheumatol Rep, 2015, 17(3):19. https://doi.org/10.1007/s11 926-014-0492-x PMID: 25761926

[3] Roddy E, Choi HK. Epidemiology of gout. Rheum Dis Clin North Am, 2014, 40(2):155-175. https://doi.org/10.1016/j.rdc. 2014.01.001 PMID: 24703341 PMCID: PMC4119792

[4] Jeon YS, Hwang DS, Hwang JM, Lee JK, Park YC. Pathological fracture of the femoral neck due to tophaceous gout: an unusual case of gout. Hip Pelvis, 2019, 31(4):238-241. https:// doi.org/10.5371/hp.2019.31.4.238 PMID: 31824879 PMCID: PMC6892900

[5] Keenan RT. The biology of urate. Semin Arthritis Rheum, 2020, 50(3 Suppl):S2-S10. https://doi.org/10.1016/j.semarthrit.2020. 04.007 PMID: 32620198 
[6] Nguyen C, Ea HK, Palazzo E, Lioté F. Tophaceous gout: an unusual cause of multiple fractures. Scand J Rheumatol, 2010, 39(1):93-96. https://doi.org/10.3109/03009740903061428 PMID: 20132078

[7] Nielsen SM, Zobbe K, Kristensen LE, Christensen R. Nutritional recommendations for gout: an update from clinical epidemiology. Autoimmun Rev, 2018, 17(11):1090-1096. https://doi.org/10. 1016/j.autrev.2018.05.008 PMID: 30213692

[8] Ragab G, Elshahaly M, Bardin T. Gout: an old disease in new perspective - a review. J Adv Res, 2017, 8(5):495-511. https:// doi.org/10.1016/j.jare.2017.04.008 PMID: 28748116 PMCID: PMC5512152

[9] Sultan AA, Whittle R, Muller S, Roddy E, Mallen CD, Bucknall M, Helliwell T, Hider S, Paskins Z. Risk of fragility fracture among patients with gout and the effect of urate-lowering therapy. CMAJ, 2018, 190(19):E581-E587. https://doi.org/10.1503/cmaj. 170806 PMID: 29759964 PMCID: PMC5953575

[10] Towiwat P, Chhana A, Dalbeth N. The anatomical pathology of gout: a systematic literature review. BMC Musculoskelet Disord, 2019, 20(1):140. https://doi.org/10.1186/s12891-0192519-y PMID: 30935368 PMCID: PMC6444644

[11] Neogi T. Gout. Ann Intern Med, 2016, 165(1):ITC1-ITC16. https://doi.org/10.7326/AITC201607050 PMID: 27380294
[12] Rock KL, Kataoka H, Lai JJ. Uric acid as a danger signal in gout and its comorbidities. Nat Rev Rheumatol, 2013, 9(1): 13-23. https://doi.org/10.1038/nrheum.2012.143 PMID: 22945591 PMCID: PMC3648987

[13] Braga TT, Forni MF, Correa-Costa M, Ramos RN, Barbuto JA, Branco P, Castoldi A, Hiyane MI, Davanso MR, Latz E, Franklin BS, Kowaltowski AJ, Camara NO. Soluble uric acid activates the NLRP3 inflammasome. Sci Rep, 2017, 7:39884. https://doi.org/10.1038/srep39884 PMID: 28084303 PMCID: PMC5233987

[14] So AK, Martinon F. Inflammation in gout: mechanisms and therapeutic targets. Nat Rev Rheumatol, 2017, 13(11):639-647. https://doi.org/10.1038/nrrheum.2017.155 PMID: 28959043

[15] Trăistaru R, Enăchescu V, Kamal D, Foarfă C, Rogoveanu O. Pseudorheumatoid disability man with chronic tophaceous gout: a case report. Rom J Morphol Embryol, 2013, 54(4): 1125-1134. PMID: 24399012

[16] Dalbeth N, Pool B, Gamble GD, Smith T, Callon KE, McQueen FM, Cornish J. Cellular characterization of the gouty tophus: a quantitative analysis. Arthritis Rheum, 2010, 62(5):1549-1556. https://doi.org/10.1002/art.27356 PMID: 20131281

\section{Corresponding authors}

Viorela Mihaela Ciortea, MD, PhD, Department of Physical and Rehabilitation Medicine, luliu Haţieganu University of Medicine and Pharmacy, 8 Victor Babeş Street, 400012 Cluj-Napoca, Romania; Phone +40721-555 466, e-mail: viorela.ciortea@yahoo.com

Kamal Constantin Kamal, Assistant Professor, MD, PhD, MSc, Department of Family Medicine, University of Medicine and Pharmacy of Craiova, 2 Petru Rareş Street, 200349 Craiova, Dolj County, Romania; Phone +40748-117 735, e-mail: kamalconstantin@gmail.com

Received: May 5, 2021

Accepted: November 22, 2021 